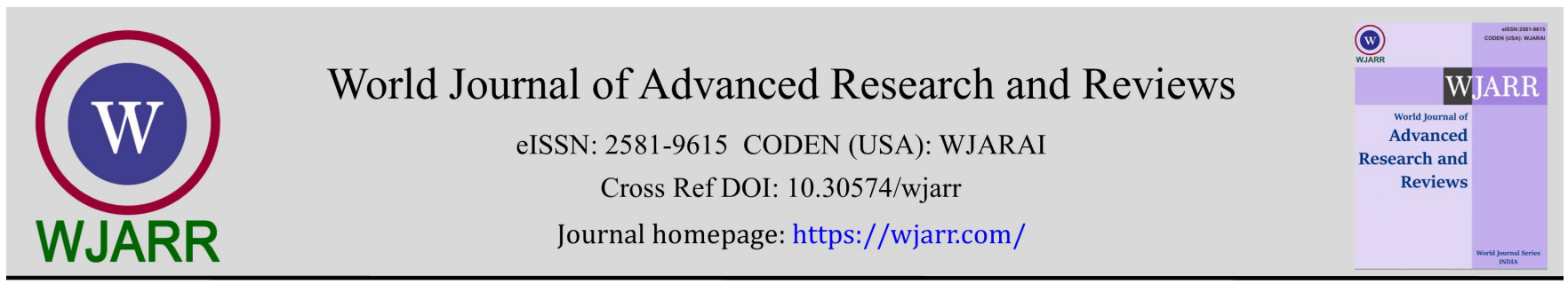

(REVIEW ARTICLE)

\title{
Radiation protection and safety in the nuclear medicine laboratory
}

\author{
Christos Iliadis 1,* , Aikaterini Frantzana 2, Kiriaki Tachtsoglou ${ }^{3}$, Ouzounakis Petros ${ }^{4}$ and Lambrini \\ Kourkouta 5
}

${ }^{1}$ Private Diagnostic Health Center of Thessaloniki, Greece.

2 General Hospital "G. Papanikolaou" Thessaloniki, Greece.

${ }^{3}$ General Hospital "G. Gennimatas" Thessaloniki, Greece.

${ }^{4}$ General Hospital of Alexandroupoli, Greece.

${ }^{5}$ Department of Nursing, Hellenic International University "DIPAE", Thessaloniki, Greece.

World Journal of Advanced Research and Reviews, 2022, 13(01), 771-774

Publication history: Received on 24 December 2021; revised on 30 January 2022; accepted on 01 Februay 2022

Article DOI: https://doi.org/10.30574/wjarr.2022.13.1.0105

\begin{abstract}
Introduction: Nuclear medicine involves the handling and storing of radioactive materials known to pose a risk to human health. The size of the exposure depends on the radionuclide, the person's activity and the type of one's work in a nuclear medicine laboratory.
\end{abstract}

Purpose: The purpose of this literature review is to investigate the contribution of radiation protection and safety measures regarding the protection of professionals' health working in the nuclear medicine laboratory, as well as patients who undergo relevant tests.

Methodology: The study material consists of recent articles on the subject mainly found in Greek and international databases as the Medline, Google Scholar and the Hellenic Academic Libraries Association (HEAL-Link).

Results: In Greece, the principles of radiation protection are defined by Law 4310/2014. Radiation protection regulations are provided for the procedures as regards the implementation of a radiation protection system in general and regulation of all issues related to the protection of the professionals' health exposed to radiation as well as for the issues arising from ionizing radiation during the activities referred to the protection of the general public by legislation.

Conclusion: The control of radiation exposure in nuclear medicine not only for health care professionals, but also for patients and the public, can be achieved effectively through many actions being crucial to this direction.

Keywords: Radiation protection; Safety; Nuclear medicine; Medicine laboratory

\section{Introduction}

Nuclear medicine involves the handling and storing of radioactive materials known to pose a risk to human health. The size of the exposure depends on the radionuclide, the person's activity and the type of one's work in a nuclear medicine laboratory. Relatively new imaging methods, including the use of positron-emitting radionuclides for PET scanning, have led to an increase in staff exposure to radioactivity. The public may also be exposed to radiation from a patient who has already been administered a radiopharmaceutical [1].

\footnotetext{
${ }^{*}$ Corresponding author: Christos Iliadis

Private Diagnostic Health Center of Thessaloniki, Greece.

Copyright (C) 2022 Author(s) retain the copyright of this article. This article is published under the terms of the Creative Commons Attribution Liscense 4.0.
} 
In this framework, radiation protection in nuclear medicine involves the control of both health care professionals' and patients' normal and potential exposure to all situations involving the use of unsealed radiation sources. Nuclear medicine professionals have a responsibility to maintain radiation safety in a way that protects both themselves and their patients from unnecessary radiation exposure [2].

As new information becomes available on advances in nuclear medicine, radiation exposure and potential hazards, it is vital that the information be shared among health care professionals, patients and the public. Given the low doses of radiation in nuclear medicine, any potential risks must be weighed against the actual benefits to the patient along with the type of imaging methodology and the cost [3].

The purpose of this literature review is to investigate the contribution of radiation protection and safety measures regarding the protection of professionals' health working in the nuclear medicine laboratory, as well as patients who undergo relevant tests.

\section{Methodology}

The study material consists of recent articles on the subject mainly found in Greek and international databases such as Medline, Google Scholar and the Hellenic Academic Libraries Association (HEAL-Link) using the keywords: radiation protection, safety, nuclear medicine. The exclusion criteria for the articles was the language, except for Greek and English. Mostly, articles and studies accessible to authors were used.

\section{General Principles of Radiation Protection}

The international unit used to measure the amount of radiation received by an individual is the millisievert (mSv). Sometimes the traditional unit, millirem (mrem), is also used, where $1 \mathrm{mSv}$ equals $100 \mathrm{mrem}$. As in all radiology professions, nuclear medicine practitioners should follow proper radiation safety practices, including optimum utilization of their time, distance, and shielding to minimize their exposure. The nuclear medicine practitioner should minimize the time spent near the radioactive patient or other radiation sources. If one can increase the distance from the radioactive patient by a factor of 2 , e.g. from 1 to $2 \mathrm{~m}$, then the radiation exposure is reduced by 4 units. Some radiopharmaceuticals, such as those containing $18 \mathrm{~F}$-fluorine deoxy glucose, emit more radioactivity due to the higher energy and number of photons emitted. However, if the practitioner can stay at least 2 meters away from the patient, the exposure is low enough for a single procedure. Urine, blood, and other biological materials obtained from patients may also contain small amounts of radioactivity for hours and possibly days after administration of the radiopharmaceutical. However, standard universal precautions taken to protect against infections also provide protection for the healthcare professional against these radioactive sources [3].

Although radiation exposure in medicine, especially in computed tomography, has been a source of great concern to the media and the general public, only high levels of ionizing radiation have been shown to have adverse health effects, particularly regarding cancer development. Computed tomography generally represents the highest exposure to radiation (27\%). Nuclear medicine (14\%), invasive radiology (8\%) and radioscopy (6\%) make up the other $50 \%$ of the ionizing radiation emitted that humans are exposed to every year. Nevertheless, there is no direct evidence that radiation levels commonly used in nuclear medicine and radiology have adverse effects on a patient's health. Nevertheless, it is considered prudent to optimize the delivery of nuclear medicine procedures, while providing a higher quality of imaging in the shortest possible time and with the lowest possible patient exposure to radiation. Some organizations, such as the Nuclear Medicine Association (EAMN), Society for Nuclear Medicine and Molecular Imaging (SNMMI), and the International Radiation Protection Commission (ICRP), have developed approaches to assessing the radiation dose to different individuals. Though the sources of public exposure are largely the same as those of nuclear medicine professionals being based on the level of acceptable risk, different dose limits apply to members of the public than to nuclear medicine professionals $[4,5]$.

In addition, public access to designated areas in hospitals and nuclear medicine departments should be limited in duration. Radiation protection of the public is achieved effectively through the proper design of the nuclear medicine departments, the restriction of access to areas where there is radiation exposure and through the safety procedures followed by the staff members of the department $[6,7,8]$.

Communicating with patients is also a prerequisite for them to understand radiation hazards, because research has shown that perceptions and conceptions of radiation hazard vary considerably, among patients, the public and even amongst physicians, nuclear medicine technologists and other workers. Thus, it is crucial that members of the nuclear 
medicine team be able to communicate effectively on these issues with referring physicians, patients and their families about nuclear medicine and radiation exposure. It is no longer enough to simply state that nuclear medicine procedures are safe. Special procedures such as leaflets can be provided in a hard copy or electronically to patients and their families to better inform them and understand the risks of radiation exposure. These should include explanations of the procedures and the clinical question that arises, as well as a brief discussion of exposure and radiation risk [3].

\section{Legislative Framework for Radiation Protection in Greece}

In Greece, the principles of radiation protection are defined by Law 4310/2014. Radiation protection regulations are provided for the procedures as regards the implementation of a radiation protection system in general and regulation of all issues related to the protection of the professionals' health exposed to radiation as well as for the issues arising from ionizing radiation during the activities referred to the protection of the general public by legislation [9].

The Greek Atomic Energy Commission (EEAE) is also the competent body that coordinates the individual dosing of personnel dealing professionally with ionizing radiation (Law 4310/2014, Government Gazette 258 / A / 08.12.2014). It is the competent authority for radiation protection ensuring that the provisions of these regulations are complied with and introduces, where necessary, additional measures to limit the individual and collective doses resulting from radiation exposure, the classification of workers and the implementation of control and monitoring measures. The individual dosing services provided by GAEC have been accredited by the ESYD according to ISO 17025, Certificate 117, for measurements of body and limb dosimeters in photon fields. Training material on employee radiation protection for operators of ionizing medical devices has already been come out $[10,11]$.

Exposed workers, for surveillance and observational purposes, are classified into the following categories: 1) Category A: exposed workers who may receive an active dose higher than $6 \mathrm{mSv}$ per year or an equivalent dose higher than 15 $\mathrm{mSv}$ per year for the lens of the eye; higher than $150 \mathrm{mSv}$ per year for skin and limbs, 2) Category B: those exposed workers are not classified in the exposed workers of category A. According to the radiation protection regulations (PD 101/2018, Government Gazette 194 / A / 20.11 .2018) Category A employees must be systematically monitored on the basis of individual measurements. The monitoring of Category B employees must be able to demonstrate at least that these employees have been correctly classified in Category B [10].

The Greek Atomic Energy Commission (EEAE) is also responsible for monitoring employees' radiation and keeping records. The protection of the population from radiation is based on an estimation of the dose that the population receives under normal or accident conditions. Surveillance is based on the population as a whole and by reference groups [11].

In addition, the 2001 Radiation Protection Regulations, adopted by Ministerial Decree 1014 (FOR) 94, set out the principles for the protection of persons, property and the environment from the dangers arising from the use of ionizing radiation. These regulations incorporate the corresponding regulations of the European Directives 96/29 / Euratom and 97/43 / Euratom of the Council. Regulations set dose limits for radiation-exposed workers and the general public. These limits apply to the production, use, processing, utilization, handling, commercial transactions, transport and disposal of natural and man-made radioactive substances, ionizing radiation equipment and any other activity that may pose a risk of ionizing radiation. The first part of the regulations sets out the basic conditions and requirements for radiological protection against activities that pose a risk of ionizing radiation. The second part refers to the conditions governing licensing procedures for all activities involving ionizing radiation. The other parts of the regulation provide a detailed description of the specific terms and requirements for radiological protection in relation to the specific activities to which they relate [9].

In addition, as a member of the International Labor Organization (ILO), Greece ratified Convention no. 115 on the Protection of Workers from Ionizing Radiation by Law 1181 of 24 July 1981. This Convention applies to all activities involving exposure of workers to ionizing radiation during their work. By Ministerial Decision 9087 (FOR) 1004 of 13 September 1996, Greece implemented Council Directive 90/641 / Euratom. Law 3850 of 11 June 2010 on the health and safety of workers also provides a framework for the health and safety of workers in the workplace. The law provides for the establishment of a committee for health and safety in each workplace, defines the responsibilities of management, safety engineers and occupational physicians, as well as the requirements for the safety and health of workers $[9,12]$. 


\section{Conclusion}

The control of radiation exposure in nuclear medicine not only for health care professionals, but also for patients and the public, can be achieved effectively through many actions being crucial to this direction. These ones include the design of facilities, the definition of workplaces in controlled and supervised areas, the individual arrangements for monitoring areas for infections and the use of personal protective equipment and protective gear on a case-by-case basis, compliance with local rules for safe handling of radiopharmaceutical products and appropriate training and education of staff. All the above constitute the basic principles of radiation protection in nuclear medicine and apply to all situations involving the handling of unsealed radiation sources from which, except for the external exposure, contamination of the staff and the work environment may also occur.

\section{Compliance with ethical standards}

\section{Disclosure of conflict of interest}

There are no conflicts of interest.

\section{References}

[1] Johnson TE, Birky BK. Health physics and radiological health. Lippincott Williams \& Wilkins. 2012.

[2] Cho SG, Kim J, Song HC. Radiation safety in nuclear medicine procedures. Nuclear medicine and molecular imaging. 2017; 51(1): 11-16.

[3] Fahey F, Goodkind A, Treves A, Grant F. Nuclear Medicine and Radiation Protection. Journal of Radiology \& Nursing. 2016; 35 (1): 5-11

[4] EANM Radiation Protection and Dose Optimization. A technologist's guide. [Internet]. 2016 [cited 2022 Jan 17] Available in: https://www.eanm.org/publications/technologists-guide/radiation-protection-doseoptimisation/

[5] Waterstram-Rich KM, Gilmore D. Nuclear Medicine and PET / CT-E-Book: Technology and Techniques. Elsevier Health Sciences. 2016.

[6] IAEA Nuclear Medicine Resources Manual. [Internet]. International Atomic Energy Agency Vienna,2006 [cited 2022 Jan 13] Available in: https://www-pub.iaea.org/MTCD/Publications/PDF/Pub1198_web.pdf

[7] IAEA (International Atomic Energy Agency). Quality Management Audits in Nuclear Medicine Practices (2nd ed) IAEA Human Health Series No. 33. Vienna. 2015.

[8] Prakash D. Nuclear Medicine: A Guide for Healthcare Professionals and Patients. Springer Science \& Business Media. 2014.

[9] OECD Nuclear Legislation in OECD and NEA Countries. Regulatory and Institutional Framework for Nuclear Activities. [Internet]. 2016 [cited 2022 Jan 19] Available in: https://www.oecdnea.org/law/legislation/greece.pdf

[10] EEAE Personal dosimetry [Internet]. 2020 [cited 2022 Jan 18] Available in: https://eeae.gr/en/services/personal-dosimetry

[11] EEAE Legislation. [Internet] 2020 [cited 2022 Jan 20] Available in: https://eeae.gr/en/regulatorydocuments/legislation

[12] EANM Quality Control of Nuclear Medicine Instrumentation and Protocol Standardisation. [Internet]. 2017 \{cited 2022 Jan 20] Available in: https://www.eanm.org/publications/technologists-guide/qualitycontrol/ 